\title{
Aspiration from the vitreous of a non-magnetic foreign body
}

\author{
HECTOR B. CHAWLA, JOSEPH A. COLEIRO, AND FRIDBERT JONASSON \\ From the Edinburgh Royal Infirmary
}

SUMMARY A non-magnetic vitreal foreign body was aspirated from over the macula through the pars plana. The surgeon guided a blunt needle towards the foreign body while watching both with the binocular indirect ophthalmoscope. His assistant's tentative suction movements with an attached syringe drew the foreign body up into the needle without risking a retinal perforation. Visual recovery was complete. It is suggested that aspiration might be the procedure of choice for such small visible intraocular fragments.

A non-magnetic foreign body in the vitreous presents a difficult and dangerous clinical dilemma, since the foreign body itself and the surgical attempt to remove it may both bring about destruction of the eye. Neubauer (1963) originally described aspiration of such a foreign body through a special spoonshaped cannula under direct vision and through an adjacent sclerotomy. More recently (Neubauer, 1975) he has advocated removal through the pars plana of freely moving vitreal foreign bodies, directly viewed with the ophthalmoscope.

We have aspirated a brass foreign body from over the macula while monitoring the intaocular manipulations with a binocular indirect ophthalmoscope. We entered the eye through the pars plana and used a standard blunt-ended 16 gauge needle attached to a standard disposable syringe. At the time we could not find any reports that might have given us the benefit of earlier experiences with such an approach. The perils of leaving a copper-rich alloy within the eye have been sufficiently well documented to convince us that surgical removal was necessary.

\section{Case report}

In August 1975 a 28 -year-old man suffered a right partial hyphaema when he was struck on the eye by a shotgun cartridge. The visual acuity, surprisingly, was $6 / 6$. No fundal view was possible, but routine $x$-ray examination was negative. After 2 days' bed rest the hyphaema subsided sufficiently to reveal a glistening 'golden' object over the macular area

Address for reprints: Dr H. B. Chawla, The Royal Infirmary, Princess Alexandra Eye Pavilion, Chalmers Street, Edinburgh EH3 9HA

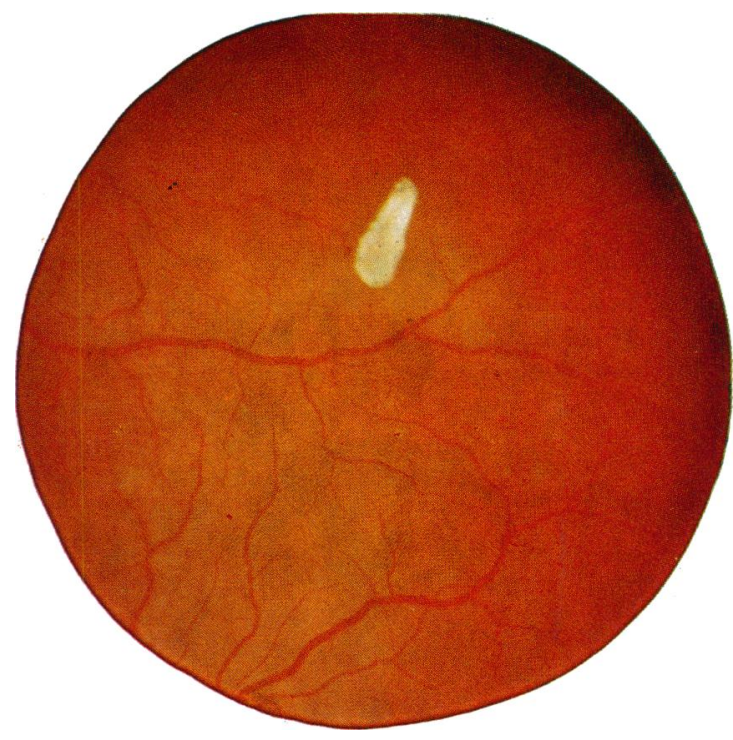

Fig. 1 'Golden' object over macular area

(Fig. 1). The patient in fact noticed its presence before we did. We presumed it to be a piece of the cartridge case, made of brass with a high copper content, and the gunmakers confirmed a level of $80 \%$ before we proceeded with the operation.

Under general anaesthesia the conjunctiva was opened along the limbus between the 7 and 11 o'clock meridians. A superficial foreign body was removed, and a penetrating entry wound was discovered just anterior to the scleral landmark of the ora serrata. Stay sutures were placed round all four rectus muscles to allow the assistant to tether 


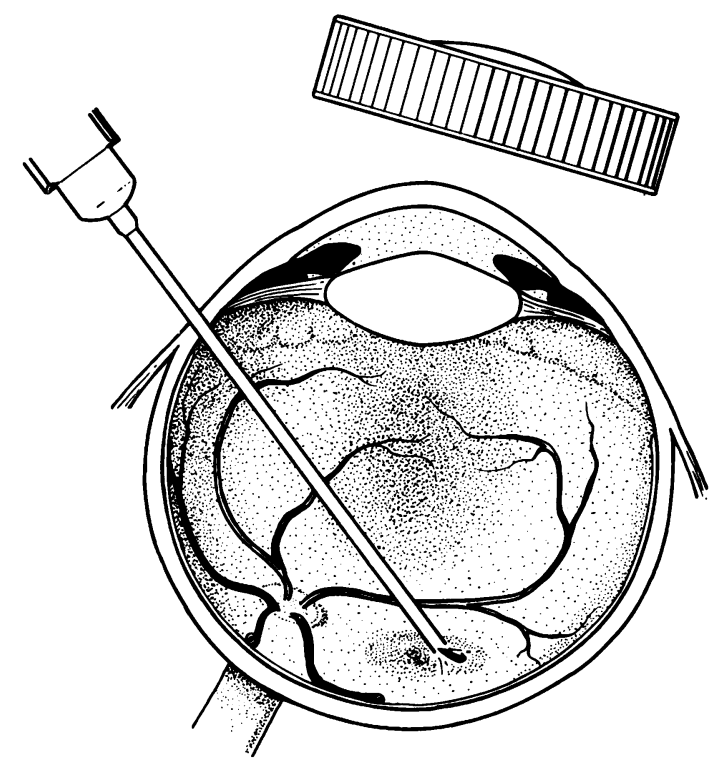

Fig. 2 Foreign body sucked into needle

the eye in any required position. The entry wound was now extended anteriorly for $1 \mathrm{~mm}$ and diathermy applied to the pars plana of the ciliary body deep to this opening. While the surgeon introduced the aspiration needle towards the macula, which he kept under continuous view with the indirect ophthalmoscope, the assistant, on his instructions, carried out tentative suction movements with the plunger of the syringe. When the blunt needle was within $2 \mathrm{~mm}$ of the foreign body, these movements sucked it into the stem of the needle (Fig. 2). The needle was removed at once, and the sclerotomy over the pars plana sutured with 5/0 PTFE coated polyester and the conjunctiva with catgut. A metal sliver $1.5 \times 0.5 \mathrm{~mm}$ was then expelled from the needle.

The patient was kept in bed for the next day or so because there had been a small haemorrhage caused by the foreign body at the point of entry. This cleared up very quickly. A short course of systemic antibiotics was given, together with local mydriatics and local steroids.

His recovery was uneventful, and 2 years later the vision in the right eye is actually better than that of the left, now being $6 / 5$. There is no evidence of vitreal disturbance, and the eye has clearly been undisturbed by this remarkably gentle invasion through the pars plana.

\section{Discussion}

No matter what the operative problem may be, surgical simplicity in both instrumentation and technique has much to commend it. In these particular circumstances, provided a foreign body is small and can be seen (larger fragments usually disappear into vitreal haemorrhage), all that is required is a routine intraocular surgical tray, a blunt-ended 16 gauge aspiration needle, a disposable syringe, and mastery of the indirect ophthalmoscope.

We are indebted to the W. H. Ross Foundation (Scotland) for their contribution towards publishing costs.

\section{References}

Neubauer, H. (1963). The removal of non magnetic foreign bodies in the vitreous by aspiration. Transactions of the Ophthalmological Societies of the United Kingdom, 83, 143-151.

Neubauer, H. (1975). Intraocular foreign bodies. Transactions of the Ophthalmological Societies of the United Kingdom, 95, 496-501. 